\title{
IS THE THRESHOLD FOR REPORTING PAIN OR THE TENDENCY TO ATTRIBUTE THE PAIN TO THE JOB LINKED TO SOCIOECONOMIC POSITION OR GENDER?
}

Ingrid Sivesind Mehlum, ${ }^{1}$ Petter Kristensen, ${ }^{1}$ Laura Punnett ${ }^{2}{ }^{1} \mathrm{NIOH}$, Oslo, Norway: ${ }^{2}$ University of Massachusetts, Lowell, USA

10.1136/oemed-2011-100382.100

Objectives Self-administered questionnaires are used in many studies. The aim of this study was to compare the threshold for reporting neck-shoulder or arm pain, and the tendency to attribute the pain to work, between subjects of high and low socioeconomic position (SEP), and between women and men. Methods A sample of 217 employed participants in the Oslo Health Study, aged 30, 40, and 45 years, who reported neckshoulder or arm pain in the past month, underwent a health examination. A criteria document was used by the physicians to establish clinical diagnoses and assess the work-relatedness of pain. We compared the odds of clinical diagnosis, using high SEP and men, respectively, as reference groups. We also measured agreement between the participants and physicians on whether pain was work-related.

Results The odds of clinical diagnoses were higher in low SEP than high SEP subjects with pain, especially for the 
elbow-forearm (OR 4.73, 95\% CI 1.64 to 13.68), and higher among women than among men, but only for neck pain (OR 2.74, 95\% CI 0.90 to 8.34). Pain reported as work-related was more frequently assessed as work-related by the physicians in low SEP than high SEP subjects (93\% vs $51 \%$ for neck-shoulder pain, and $89 \%$ vs $70 \%$ for arm pain), and in men.

Conclusions The results suggest that the threshold for reporting pain is higher in low SEP subjects and among women, and that physicians are more likely to agree about work etiology in low SEP subjects and men. 